\title{
Nuclear level density predictions
}

\author{
Dorel Bucurescu ${ }^{1, a}$ and Till von Egidy ${ }^{2}$ \\ ${ }^{1}$ Horia Hulubei National Institute for Physics and Nuclear Engineering (IFIN-HH), Bucharest, Romania \\ ${ }^{2}$ Technische Universität München, Garching, Germany
}

\begin{abstract}
Simple formulas depending only on nuclear masses were previously proposed for the parameters of the Back-Shifted Fermi Gas (BSFG) model and of the Constant Temperature (CT) model of the nuclear level density, respectively. They are now applied for the prediction of the level density parameters of all nuclei with available masses. Both masses from the new 2012 mass table and from different models are considered and the predictions are discussed in connection with nuclear regions most affected by shell corrections and nuclear structure effects and relevant for the nucleosynthesis.
\end{abstract}

\section{Introduction}

The nuclear level density (NLD), as basic information on the nuclear structure at low and high excitation energies, is a key quantity in many domains. It is needed for calculations of compound nucleus reactions and also for the research on exotic nuclei (far from stability). The investigation of the nucleosynthesis requires its knowledge for many unknown nuclei. In this contribution we address the problem of the prediction of nuclear level densities for such nuclei by extrapolations based on simple phenomenological NLD models and global nuclear mass calculations.

\section{The models}

Phenomenological NLD models are frequently used in parallel to improved microscopic models due to their simple application. In several papers [1-3] we propose the use of simple models such as the Back Shifted Fermi Gas (BSFG) and the Constant Temperature (CT) model for the level densities at least up to the neutron binding energy. We give in the following the prominent features of this type of description. The NLD formulas for the two models

$$
\rho_{B S F G}=\frac{e^{2 \sqrt{a\left(E-E_{1}\right)}}}{12 \sqrt{2} a^{1 / 4}\left(E-E_{1}\right)^{5 / 4}},
$$

and

$$
\rho_{C T}=\frac{1}{T} e^{\left(E-E_{0}\right) / T},
$$

respectively, have each two free parameters, $a$ and $E_{l}$, and $T$ and $E_{0}$, respectively. They were fitted to discrete energy levels at low energies and resonance spacings for

\footnotetext{
a Corresponding author: bucurescu@tandem.nipne.ro
}

a total of 310 nuclei between ${ }^{19} \mathrm{~F}$ and ${ }^{251} \mathrm{Cf}$ (all listed in $[1,2])$. Simple formulas were found which describe these empirical parameters using only quantities taken from nuclear mass tables [1-3]. They can be used to extrapolate to nuclei for which the NLD parameters cannot be derived experimentally. For example, in ref. [2] the following formulas have been proposed:

$$
\begin{gathered}
a=\left(0.199+0.0096 S^{\prime}\right) A^{0.869} \\
E_{1}=-0.381+0.5 P a^{\prime} \\
\mathrm{T}=A^{-2 / 3}\left(0.0597+0.00198 S^{\prime}\right)^{-1} \\
E_{0}=-1.004+0.5 P a
\end{gathered}
$$

Here, $S^{\prime}=S+0.5 P a^{\prime}$, where $S=M_{\text {exp }}-M_{\text {weiz }}$ is the shell correction. $M$ denotes the mass (with $M_{\text {weiz }}$ calculated with the Weizsäcker-type formula of [4]). $P a$ ' is the socalled deuteron pairing, defined as

$$
P a^{\prime}=[M(A+2, Z+1)-2 M(A, Z)+M(A-2, Z-1)] / 2
$$

The empirical parameters of the formulas (1) to (4) were deduced with a spin-cutoff parameter of the spin distribution function parameterized as $[2,3]$ :

$$
\sigma^{2}=0.391 A^{0.675}\left(E-0.5 P a^{\prime}\right)^{0.312}
$$

whereas in ref. [1] slightly different formulas (instead of (1) to (4)) were deduced by using a different definition of $\sigma$ and of the sign of the deuteron pairing. In refs. $[1,2]$ the experimental masses for the 310 nuclei were taken from the mass tables of 2003 [5], but since the experimental masses of these nuclei did not significantly change, these results are also valid for the new mass tables of 2012 [6]. 
One salient feature of our formulas is the use of the 'deuteron pairing' (5) both in the shell correction $S$ ' and in the back-shift parameters $E_{1}, E_{0}$, and the spin-cutoff parameter $\sigma$. Figure 1 shows that this useful quantity distinguishes between even-even, odd-A, and odd-odd nuclei, and roughly compares to the average pairing gap value $\Delta=12 / A^{1 / 3}$. In refs. [1-3] one can also see that formulas (2) and (4) adequately describe some details of the behaviour of $E_{1}, E_{0}$ with the mass number.

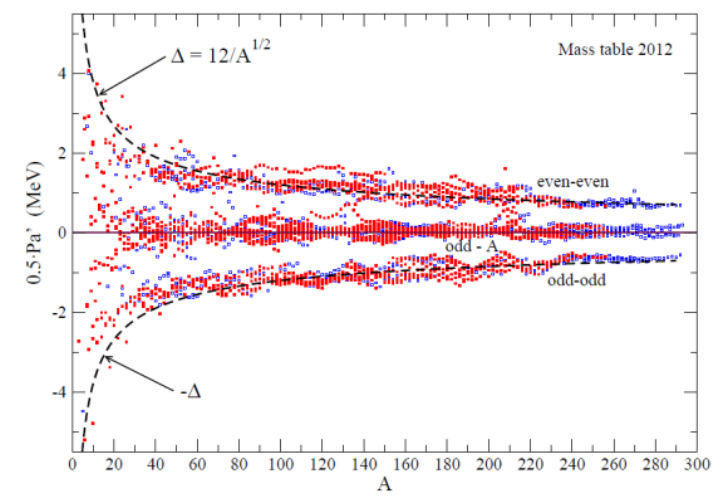

Figure 1. Deuteron pairing $\mathrm{Pa}$ ' calculated for all nuclei listed in mass table 2012 [6]. The colours red and blue distinguish nuclei with measured and extrapolated masses, respectively.

One should also emphasize that the factor 0.5 appearing in both $S^{\prime}$ and formulas (2) and (4) was deduced by the fit. Another remarkable aspect is the proportionality of the nuclear temperature parameter $T$ with $A^{-2 / 3}$; this fact suggests that the number of degrees of freedom determining the nuclear temperature is proportional to the nuclear surface.

\section{Prediction of nuclear level densities}

The nuclear masses which determine, according to our formulas, the NLD at energies at least up to the neutron binding energy, are quantities that embed complex information on the nuclear structure. Indeed, the rather irregular behavior with $N, Z$ of the mass surface is known to reveal important details such as shell and subshell closures, as well as different types of collective nuclear structure paradigms and transitions between them.

The 310 nuclei that were used to deduce formulas (1) to (4) for the NLD parameters are nuclei close to stability. However, they span nuclear regions that are dominated by various nuclear structure phenomena, like closed shells, well deformed nuclei, transitions from spherical to deformed nuclei, etc. Thus, we believe that the deduced dependencies on nuclear masses include all essential information on the general evolution of the nuclear structure. For this reason, these equations can be proposed to predict the level density parameters at least for the nuclei with measured masses. Therefore, in the following we investigate the main features of this type of extrapolation. Figure 2 shows the $a$ parameter of the BSFG model, as determined from eq. (1) for all nuclei listed in mass table 2012 [6] for which the deuteron $\mathrm{Pa}$ ' could be determined by eq. (5) from the listed masses. We shall discuss only this important parameter of the
BSFG model because the $T$ paramater of the CT model is known to be well correlated with $a[2,7]$.

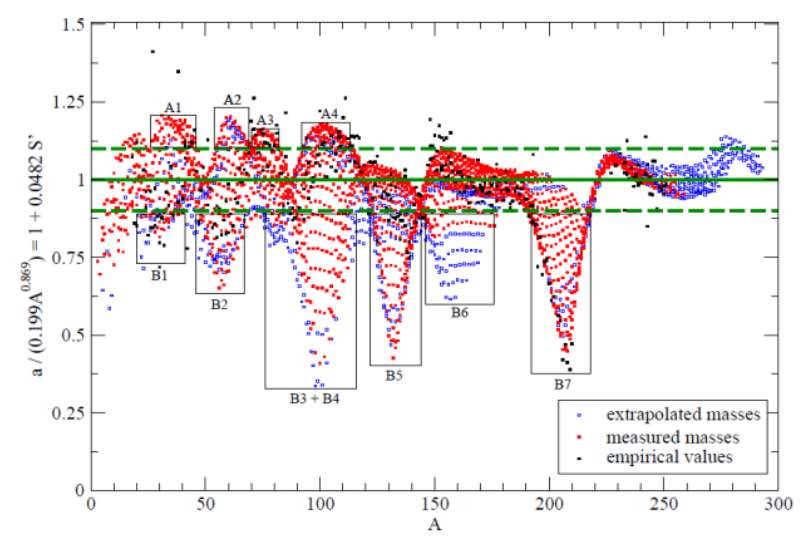

Figure 2. Normalized $a$ parameter calculated with eq. (1) for nuclei from mass table 2012 [6]. The parameter $a$ has been divided by $\left(0.199 A^{0.869}\right)$ therefore it reduces to $\left(1-0.0482 S^{\prime}\right)$. Black symbols are the experimental (fitted) empirical values. The two thick dashed lines show the limits of $+10 \%$ and $-10 \%$ deviation from 1. Boxes labelled A1, A2, ... show nuclei where the shell correction $-0.0482 \mathrm{~S}^{\prime}$ is positive and larger than $10 \%$, while the boxes labelled with $\mathrm{B} 1, \mathrm{~B} 2, \ldots$ indicate nuclei with shell corrections negative and larger than $10 \%$.

One can see that for a large number of nuclei the shell correction $-0.0482 S^{\prime}$ is within $\pm 10 \%$ of the average (uncorrected) value $0.199 \mathrm{~A}^{0.869}$. The labelled extrema are regions, generally related to shell closures, where the shell correction is bigger. A better representation is the contour map in Fig. 3, where shell corrections of different magnitudes appear as regions in the $(N, Z)$ plane.

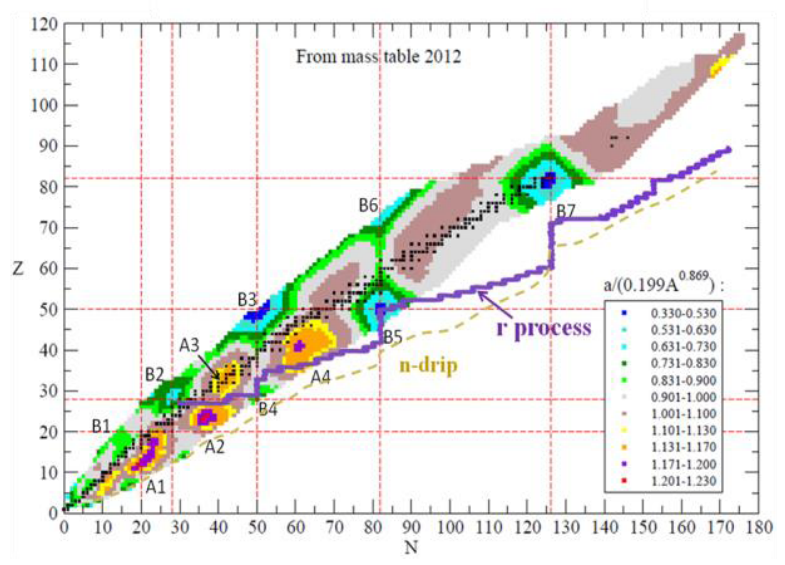

Figure 3. The normalized $a$ parameter from Fig. 2 (for nuclei from the 2012 mass table), represented in the (N,Z) plane. An estimated neutron-drip line and an assumed average path for the r-process are also shown.

The extrema from Fig. 2 appear either as 'mountains' (yellow-red, with $a$ values larger than the average value $0.199 \mathrm{~A}^{0.869}$ by more than $10 \%$ ), or as 'lakes (blue, with $a$ values smaller than the average by more than $10 \%)$. Most 
of the nuclei (2031, almost two thirds of the 3348 tabulated in [6]) have $a$ values resulting from shell corrections within $\pm 10 \%$ of the average value - they are represented by the 'plane' (light grey and light brown colors) around the valley of stability. Thus, one may say that for about 2000 nuclei listed in the 2012 mass table, even the simple prediction $a=0.199 \mathrm{~A}^{0.869}$ may be taken as having a reasonably small error. Large shell corrections, leading to smaller $a$-values (smaller level densities) are predicted especially at the magic numbers.

Many nuclei of interest lie outside the region covered by the nuclei with measured (or reasonably extrapolated) masses, especially those in the neutron-rich region which are important for the astrophysical r-process (Fig. 3). To predict NLD parameters for such nuclei with the same formulas (such as (1) to (4)) the only way is to use nuclear masses predicted by model calculations. During the past decades there have been many global mass model calculations with increasing precision in describing the known nuclear masses. They largely fall into three categories, (i) macroscopic-microscopic models, like the finite-range droplet model (FRDM) (ref. [8], and recently with a more accurate adjustment [9]), (ii) microscopic self-consistent mean-field HFB calculations (e.g., ref. [10]), and (iii) new WeizsäckerSkyrme mass formulas (e.g. ref. [11]). Because they fit the known masses, their predictions are rather similar in the region covered in Fig. 3, but they often quickly diverge outside this region and considerably differ in the region of the drip lines. It was recently shown that there is practically no correlation between the accuracy of the description of masses by a model and its predictive power [12], therefore by using more such models and comparing their predictions for the NLD parameters, one can only get a qualitative idea where there are the strongest deviations from the average $A$-dependence $a=0.199$ $\mathrm{A}^{0.869}$. As an example, Fig. 4 shows the contour map of $a$ predicted by masses of [8].

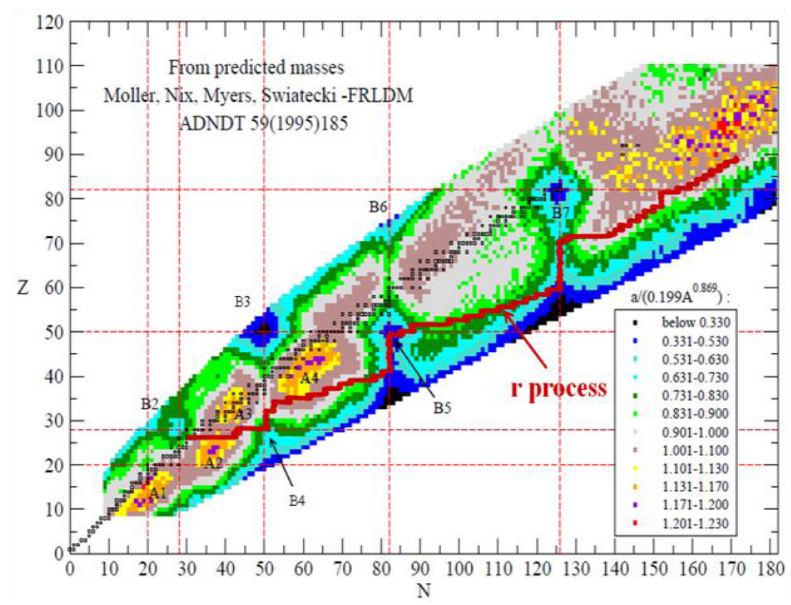

Figure 4. Same as Fig. 3, but for 6400 nuclei with masses predicted by the FRLD model [8].

One observes the similarity with Fig. 3 in the region of the nuclei with known masses. The shell corrections increase towards the neutron-drip line and are maximum at the closed shells. We also note that the A-type regions (with $a$-values larger than the average) are in the proximity of regions of interesting nuclear structure properties. For example, A2 is close to the 'island of inversion' at $Z=25$, whereas $\mathrm{A} 4$ is in the region of sudden onset of deformation of the nuclei with $\mathrm{A} \approx 100$. We have also investigated the predictions based on the microscopic HFB model known as HFB21 [10]. They are very similar to those from Fig. 4 for nuclei with known masses, but increasingly different for n-rich nuclei, the HFB model predicting a more accentuated shell correction towards the neutron-drip line.

In conclusion, the formulas proposed [1-3] for the parameters of the BSFG and CT models of the level densities provide a simple basis for the extrapolation to nuclei far from stability, by using measured nuclear masses [6] or, for more exotic nuclei, masses predicted by different models. For the later, however, the predictive power is largely unknown, as is the case for the nuclear masses themselves.

Support within the Romanian UEFISCDI Project Nr. PNII-ID-PCE-2011-3-0140 is acknowledged.

Note: files with the $a, E_{1}, T, E_{0}$ parameters calculated by eqs. (1) - (4) for the 2012 mass table [6] (3213 nuclei) and the FRLD model [8] (6641 nuclei), respectively, can be found at http://tandem.nipne.ro/ dbucurescu .

\section{References}

1. T. von Egidy, D. Bucurescu, Phys. Rev. C 72, 044311 (2005); C 72, 049901(E) (2006)

2. T. von Egidy, D. Bucurescu, Phys. Rev. C 80, 05431 (2009)

3. T. von Egidy, D. Bucurescu, IOP J. Phys. Conf. Ser. 338, 012028, (2012)

4. J.M. Pearson, Hyperfine Interactions 132, 59 (2001)

5. G. Audi, A.H. Wapstra, C. Thibault, Nucl. Phys. A 729, 337 (2003)

6. M. Wang, G. Audi, A.H. Wapstra, F.G. Kondev, M. MacCormick, X.Xu, B. Pfeiffer, Chin. Phys. C36, 1603 (2012)

7. D. Bucurescu, T. von Egidy, Phys. Rev. C 72, 067304 (2005)

8. P. Möller, J.R. Nix, W.D. Myers, W.J. Swiatecki, At. Data Nucl. Data Tables 59, 185 (1995)

9. P. Möller et al., Phys. Rev. Lett. 108, 052501 (2012)

10. S. Goriely, N. Chamel, J.M. Pearson, Phys. Rev. C 80, 035804 (2010); mass table for HFB21 from http://www.astro.ulb.ac.be/pmwiki/Brusslib/Hfb17sh $\underline{\mathrm{ells}}$

11. M. Liu, N. Wang, Y.G. Deng, X.Z. Wu, Phys. Rev. C 84, 01433 (2011)

12. A. Sobiczewski, Yu.A. Litvonov, Phys. Rev. C 90, 017302 (2014) 
